\title{
Photonic Wannier-Stark Ladder from Coupled Electromagnetic Cavities
}

\author{
Shahzad Anwar, Sucheng Li, Weixin Lu, and Bo Hou \\ College of Physics, Optoelectronics and Energy and Collaborative Innovation Center of Suzhou Nano Science and Technology, \\ Soochow University, 1 Shizi Street, Suzhou 215006, China
}

Correspondence should be addressed to Bo Hou; houbo@suda.edu.cn

Received 8 March 2015; Accepted 1 May 2015

Academic Editor: Weiren Zhu

Copyright (c) 2015 Shahzad Anwar et al. This is an open access article distributed under the Creative Commons Attribution License, which permits unrestricted use, distribution, and reproduction in any medium, provided the original work is properly cited.

\begin{abstract}
We have investigated the photonic Wannier-Stark ladder in the system of coupled electromagnetic cavities, which consists of a stack of metallic plates structured with subwavelength apertures and where the tilted potential effect is mimicked by imposing the gradient variation of refractive index. Making an analogy to its quantum counterpart and assuming the translational property of its solutions, we have shown the photonic ladder has the eigenenergies, that is, frequencies, in a geometrical series. Within the approximation of small gradient, the ladder states manifest the equidistant frequency spacing in the spectrum. By both analytical derivation and numerical simulation, we have illustrated the geometrically progressed energies of the photonic Wannier-Stark ladder.
\end{abstract}

\section{Introduction}

A lot of similar physical properties have been revealed between electrons in a solid and photons in a periodic structure [1]. Besides band gap, another fascinating example is the concepts of Wannier-Stark ladder (WSL) and Bloch oscillation (BO) [2]. When a solid is biased by a static electric field, the electron eigenstates do not belong to the extended Bloch states because the linear potential imposed by the static electric field breaks the translational symmetry of the periodical crystal potential but becomes the WSL which is a family of localized wavefunctions with equidistant energies in the energy spectrum. The Bloch oscillation, in which the electrons driven by the DC bias oscillate periodically in semiclassical picture, is just the time domain counterpart of the quantum-mechanical WSL. Due to the potential applications toward high speed electronics, both WSL and BO have been theoretically investigated and experimentally observed in the semiconductor superlattices [3]. In contrast, WSL and BO in natural crystals are difficult to observe because the coherence of electron wave packets is very fragile to various decoherence processes, for example, disorder, in the crystals.

Recently, the two concepts advance to electromagnetic (EM) waves in an analogy way, driven by the physical similarity of wave systems and the phenomenological robustness of classical waves [4]. For EM waves, the gradient refractive index or thickness of the dielectric layers introduced to a photonic structure plays the same role as the DC field in electronic cases and gives rise to both the photonic WSL in the frequency domain and the photonic $\mathrm{BO}$ in the time domain [5-7]. Besides the conventional photonic crystals [811], the microcavity systems from metamaterial with negative index have been shown to display the photonic BO [12-16]. In addition, the $\mathrm{BO}$ can be demonstrated equivalently inside the transversely coupled waveguide arrays undergoing the gradient modulation along the transverse direction $[17,18]$ and manifests the rich physics of wave propagation, such as quasi and fractional versions $[19,20]$. More recently, the WSL has also been observed in acoustic/elastic systems [21-24].

In this work, we have investigated the photonic WSL in the system of coupled EM cavities, where the biased potential effect is mimicked by imposing the gradient variation of refractive index. A numerical model is constructed, using a stack of metallic plates patterned with subwavelength apertures. By calculating the transmission, we have observed that the transmission peaks of the ladder states have the equidistant frequency spacing in the spectrum, that is, their frequencies (eigenenergies) showing an arithmetical series, 


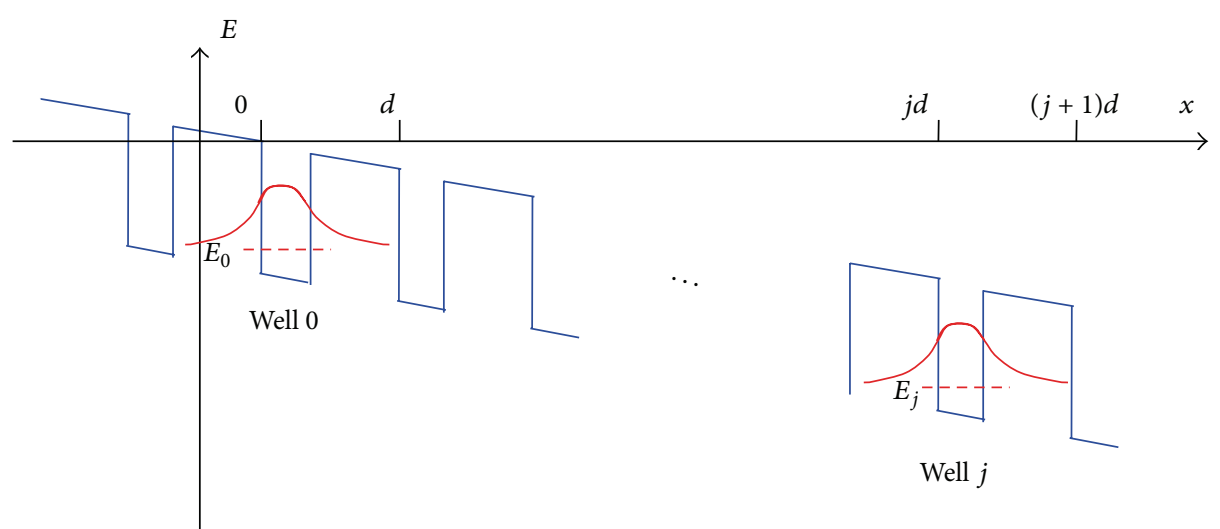

(a)

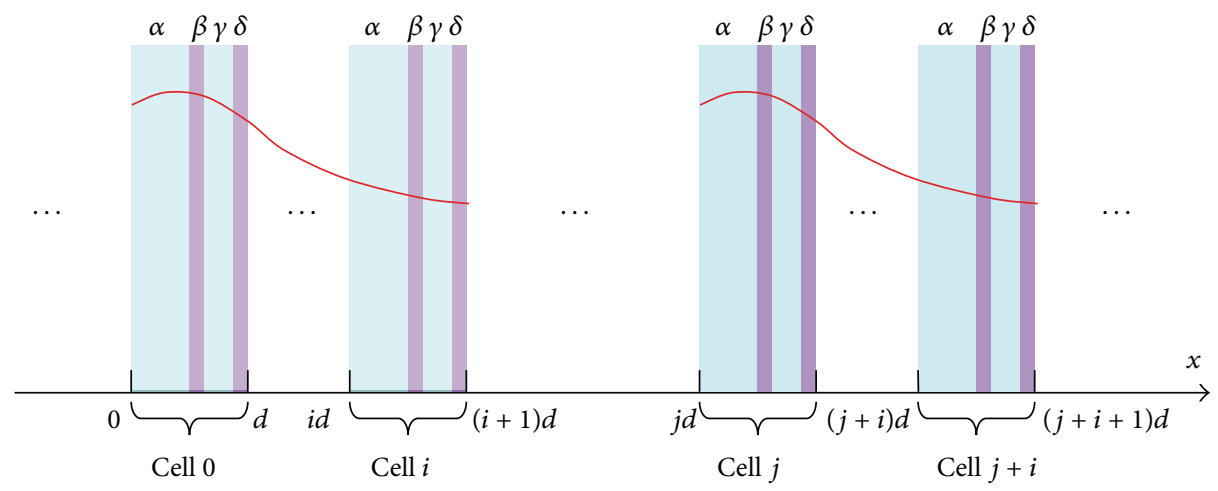

(b)

Figure 1: (a) The Kronig-Penney potential (coupled quantum wells) titled by a static electric field, where $d$ is the spatial periodicity between the neighboring wells and the red line depicts schematically the quantum WSL state localized in the different well. (b) The one-dimensional coupled EM cavities, that is, "photonic wells," with increasing the refractive indexes of the dielectric layers, $\alpha, \beta, \gamma$, and $\delta$, along the $x$-direction.

in the case of small gradient. Specifically, the frequencies of the peaks evolve into a geometrical series in the case of large gradient, which points out the generalized photonic WSL with geometrically progressed energies.

\section{Materials and Methods}

We start with the quantum WSL problem. Consider a particle of mass $m$ and charge $q$ moving in the simplified crystal potential of spatial periodicity $d$ (Kronig-Penney model [25]) tilted by a static electric field $\xi$, as depicted in Figure 1(a). For the quantum system, the Schrodinger equation has the following expression:

$$
-\frac{\hbar^{2}}{2 m} \frac{d^{2} \psi(x)}{d x^{2}}+V(x) \psi(x)=E \psi(x),
$$

where $\psi(x)$ is the wavefunction, $E$ is the eigenenergy, and the position-dependent potential has the property $V(x)=$ $V(x+d)+q \xi d$. When an electron moves in the tilted periodic potential, it will localize somewhere due to the translational symmetry breaking. Assume such a state $\psi(x)$ localized near well 0 and with energy $E_{0}$, and then the above equation reads

$$
-\frac{\hbar^{2}}{2 m} \frac{d^{2} \psi(x)}{d x^{2}}+V(x) \psi(x)=E_{0} \psi(x) .
$$

Change the variable $x$ to $x-j d$ ( $j$ being an integer), and we obtain

$$
\begin{aligned}
& -\frac{\hbar^{2}}{2 m} \frac{d^{2} \psi(x-j d)}{d x^{2}}+V(x-j d) \psi(x-j d) \\
& \quad=E_{0} \psi(x-j d) ;
\end{aligned}
$$

that is,

$$
\begin{aligned}
& -\frac{\hbar^{2}}{2 m} \frac{d^{2} \psi(x-j d)}{d x^{2}}+[V(x-j d)-q \xi j d] \psi(x-j d) \\
& \quad=\left(E_{0}-q \xi j d\right) \psi(x-j d),
\end{aligned}
$$

or

$$
\begin{aligned}
& -\frac{\hbar^{2}}{2 m} \frac{d^{2} \psi(x-j d)}{d x^{2}}+V(x) \psi(x-j d) \\
& \quad=E_{j} \psi(x-j d), \quad \text { with } E_{j}=E_{0}-q \xi j d .
\end{aligned}
$$

Therefore, a new solution $\psi(x-j d)$ is localized near the well $j$ and has the energy $E_{j}$. It is seen that a family of such states can be obtained with the consistent wavefunctions $\psi$, except that their locations translate with respect to each other. The family of states is just the WSL where their eigenenergies compose 
an arithmetical progression, $E_{j}=E_{0}-q \xi j d$, with common difference $q \xi d$.

The photonic WSL has been proved in one-dimensional coupled EM cavities $[5,6]$, where a ramp-up of the refractive index along the stacking direction is used to mimic the effect of the electric field $\xi$ in the quantum case, as illustrated in Figure 1(b). Here, we reformulate the EM problem in the same style as solving the quantum system. For explicitness and simplification, the individual cavity is assumed including four dielectric layers, and the solution also holds for the cavity with more or less layers. The Helmholtz wave equation for the photonic system is written as

$$
\frac{d^{2}}{d x^{2}} F(x)+n^{2}(x) \frac{\omega^{2}}{c^{2}} F(x)=0,
$$

where the refractive index profile

$$
n(x)= \begin{cases}n_{i}^{\alpha}, & x \in\left[i d, i d+L^{\alpha}\right), \\ n_{i}^{\beta}, & x \in\left[i d+L^{\alpha}, i d+L^{\alpha}+L^{\beta}\right), \\ n_{i}^{\gamma}, & x \in\left[i d+L^{\alpha}+L^{\beta}, i d+L^{\alpha}+L^{\beta}+L^{\gamma}\right), \\ n_{i}^{\delta}, & x \in\left[i d+L^{\alpha}+L^{\beta}+L^{\gamma},(i+1) d\right),\end{cases}
$$

with the integer $i$ specifying the cavity cell and the Greek alphabet specifying the layer, and the cavity length $d=L^{\alpha}+$ $L^{\beta}+L^{\gamma}+L^{\delta}$ with $L^{\alpha}, L^{\beta}, L^{\gamma}$, and $L^{\delta}$ being the thicknesses of the four layers, $\alpha, \beta, \gamma$, and $\delta$, respectively. Following $n(x)$, the photonic state, $F(x)$, is supposed to be sectioned. Similar to the quantum case, we hypothesize a solution, $F(x)$, which is centralized somewhere, to say near cell 0 , and has the photon energy $\hbar \omega_{0}$ and the field function:

$$
\begin{aligned}
& F(x) \\
& = \begin{cases}F_{i}^{\alpha}(x), & x \in\left[i d, i d+L^{\alpha}\right), \\
F_{i}^{\beta}(x), & x \in\left[i d+L^{\alpha}, i d+L^{\alpha}+L^{\beta}\right), \\
F_{i}^{\gamma}(x), & x \in\left[i d+L^{\alpha}+L^{\beta}, i d+L^{\alpha}+L^{\beta}+L^{\gamma}\right), \\
F_{i}^{\delta}(x), & x \in\left[i d+L^{\alpha}+L^{\beta}+L^{\gamma},(i+1) d\right),\end{cases}
\end{aligned}
$$

where the cell number $i$ may range within the integers $[-M, M]$, depending on the localizing degree. In fact, the positive integer $M$ characterizes the localization scale of the solution $(M \rightarrow 0$ representing a state localized inside only one cell and $M \rightarrow \infty$ meaning the weak localization). Without losing generality, the solution in the region $x \in$ $\left[i d+L^{\alpha}, i d+L^{\alpha}+L^{\beta}\right)$ satisfies

$$
\frac{d^{2}}{d x^{2}} F_{i}^{\beta}(x)+\left(n_{i}^{\beta}\right)^{2} \frac{\omega_{0}^{2}}{c^{2}} F_{i}^{\beta}(x)=0 .
$$

Changing the variable, we obtain

$$
\frac{d^{2}}{d x^{2}} F_{i}^{\beta}(x-j d)+\left(n_{i}^{\beta}\right)^{2} \frac{\omega_{0}^{2}}{c^{2}} F_{i}^{\beta}(x-j d)=0,
$$

or

$$
\frac{d^{2}}{d x^{2}} F_{i}^{\beta}(x-j d)+\left(n_{j+i}^{\beta}\right)^{2} \frac{\omega_{j}^{2}}{c^{2}} F_{i}^{\beta}(x-j d)=0,
$$

where $n_{j+i}^{\beta} \omega_{j}=n_{i}^{\beta} \omega_{0}$ and $x \in\left[(j+i) d+L^{\alpha},(j+i) d+L^{\alpha}+L^{\beta}\right)$. With applying to the other layers and cells, we can see that the new field function, $F(x-j d)$, its segmented expressions being $F_{i}^{\alpha, \beta, \gamma, \delta}(x-j d)$, actually represents another state which centralizes near the cell $j$ and has the energy $\hbar \omega_{j}$, where $\omega_{j}=n_{i} \omega_{0} / n_{j+i}$. For the strongly localized photonic state $(M \rightarrow 0)$, for example, the one which allows the tight binding description of the coupling effect between the EM cavities, the energy relation $n_{i} \omega_{0}=n_{j+i} \omega_{j}$ can be simplified as $n_{0} \omega_{0}=n_{j} \omega_{j}$. This relation can also be justified directly from (6), if the photonic WSL takes a series of solutions which are spatially translational to each other, like the quantum case.

It can be proved that a geometric series, $n_{i}=n_{0} q^{i}$ and $\omega_{j}=\omega_{0} / q^{j}$ ( $q$ being the common ratio), meets exactly the relation $n_{i} \omega_{0}=n_{j+i} \omega_{j}$ or $n_{0} \omega_{0}=n_{j} \omega_{j}$. Particularly, in the case of $q=1+\eta$ with the approximation $\eta \ll 1, n_{i}=n_{0}(1+\eta)^{i} \approx$ $n_{0}+i n_{0} \eta$ and $\omega_{j}=\omega_{0}(1+\eta)^{-j} \approx \omega_{0}-j \omega_{0} \eta$, where $\omega_{0} \eta$ is identified as Bloch oscillation frequency $\omega_{B}$ which manifests the equidistant frequency spacing in spectra.

\section{Results and Discussion}

In order to illustrate the photonic WSL states in a specific system, we employ a metallic plate patterned with narrow $\mathrm{H}$-fractal slits as EM cavity. The resonant state supported by the exotic fractal pattern has been found to be responsible for transmission enhancement of EM wave through such metallic plates [26]. When a number of the metallic plates with $\mathrm{H}$-fractal slits are cascaded, their resonant modes will couple together; the transmission bands would be developed in a way analog to the formation of miniband in the superlattice and furthermore comply with the photonic tight binding description [27]. Note that our fractal slits should not be considered as a sole candidate, while all other shaped apertures supporting the local resonance are applicable, too.

The coupled cavity system is 8 stacked metallic plates on which a periodic array of fractal slits was cut with lattice constant smaller than the relevant incident wavelength to avoid the grating effect, illustrated schematically in Figure 2(a). The unit cell of the array includes a five-level $\mathrm{H}$-fractal slit structure where the longest slit is $10 \mathrm{~mm}$ and the width of each slit is $0.8 \mathrm{~mm}$ [26], and the plates have a thickness of $3 \mathrm{~mm}$ and a spacing of $9 \mathrm{~mm}$. Numerically, we employ a commercial finite-difference time-domain (FDTD) software package to investigate the microwave spectra [28]. The microwave polarization was such that the electric field is perpendicular to the shortest slits of the fractal pattern. In the simulation, we assume periodic boundary condition in the transverse direction and perfect conductor approximation for metal.

To impose a tilted potential effect, we embed the plates in the dielectrics, which have the geometrically increasing refractive index, $n_{j}=(1+d n)^{j}$, where the integer $j$ denotes the plate number from 0 to 7 and the varying quantity $d n$ tunes the magnitude of the common ratio of the geometrical series. Each structured plate is embedded inside the dielectric in a symmetrical way, as illustrated in Figure 2(b). 


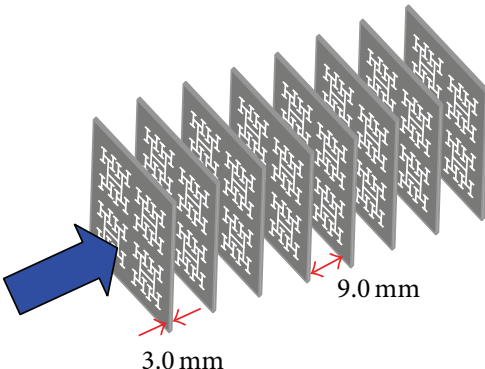

(a)

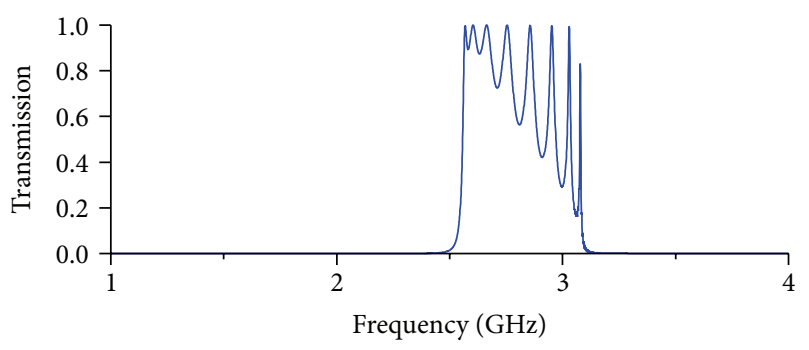

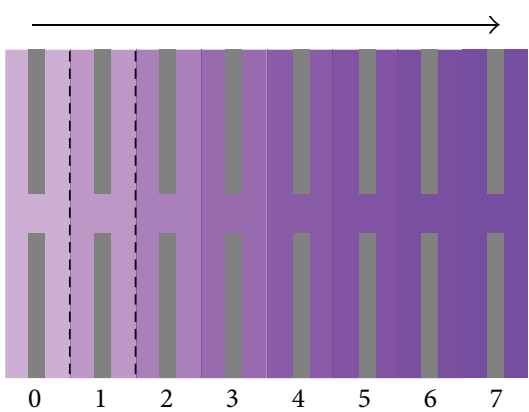

(b)

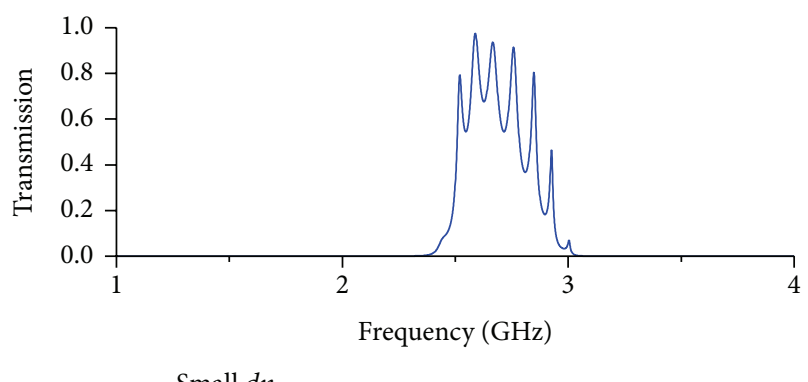

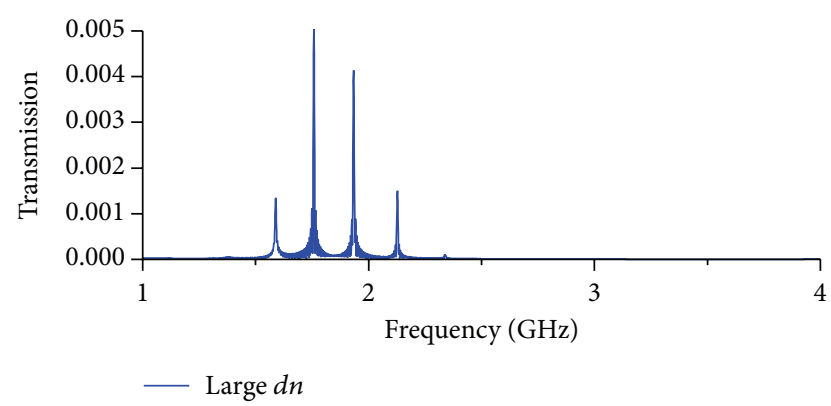

(c)

FIGURE 2: (a) The 8 stacked metallic plates on which a periodic array of fractal slits was generated. In the schematic picture, the plate has $2 \times 2$ unit cells, each of which is a five-level $\mathrm{H}$-fractal slit. (b) The 8 plates are embedded, respectively, inside 8 dielectrics, which have the refractive index, $n_{j}=(1+d n)^{j}$, where $j$ takes the denoted numbers. The index gradient is along the propagation direction, as pointed by the arrow. The dash lines depict a single EM cavity cell composed of a structured plate and a homogeneous dielectric. (c) The simulated transmissions at normal incidence of microwave, where three cases, no, small, and large gradient of refractive index, are considered.

When $d n=0$, which is referred to as the periodic case because there is no refractive index gradient, the simulated result shows that a passband is formed between 2.5 and $3.1 \mathrm{GHz}$ in the same physics as the miniband in the semiconductor superlattice, seeing the upper curve in Figure 2(c). The peaks originate from the coupling of the resonant modes in the plates at different phase delay, analogous to the development of energy band (or miniband) in crystal (or superlattice) within the tight binding description [25, 27]. It is seen that the peaks are more crowded at the two band edges than in the middle region, which is the typical density-ofstate characteristics of the miniband.

After a small variation, $d n=0.01$, is introduced, the magnitudes and positions of the peaks change drastically, seeing the middle curve in Figure 2(c). The underlying resonance states, responsible for transmission, become localized somewhere in the stacking direction. For example, the peak at the lower band edge has degraded into a spectral shoulder $(\sim 2.45 \mathrm{GHz})$ with low transmission $(\sim 10 \%)$. This is because the fractal slits which support the peak are being located at the far end plate of the stacking system, where the refractive index is highest, and the other plates with the higher resonance frequencies behave like the potential barrier. Consequently, the incident wave has to tunnel through them to excite the eigenmode of the farthest one, similar to the Zener tunneling $[23,24]$. Likewise, the situation also occurs at the upper band edge, the tiny peak at $3.0 \mathrm{GHz}$. Note that the peaks' spacing in frequency begin to appear even.

While a large variation, $d n=0.1$, is considered, an obvious downshift of the miniband region is seen in the spectrum, as plotted in Figure 2(c), originating from the overall increasing in the refractive index. At the same time, the transmission magnitude is suppressed significantly, as the titled potential is much steeper and the states have become 


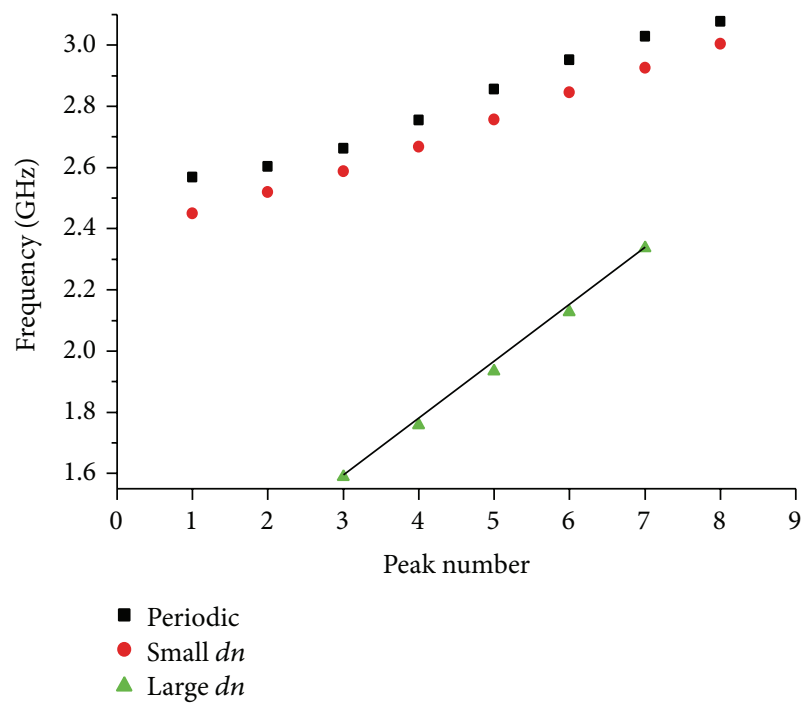

(a)

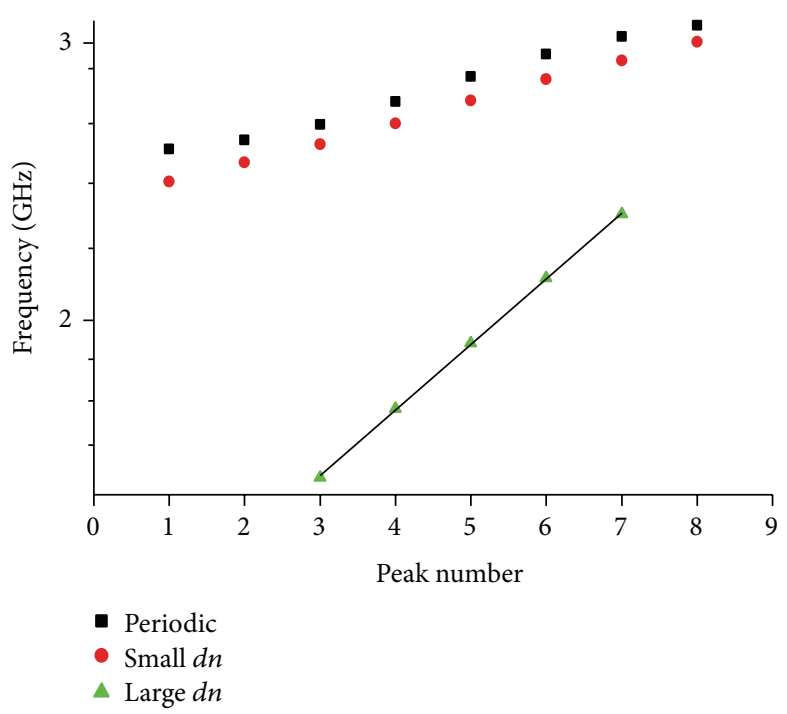

(b)

Figure 3: (a) The peak frequencies in the three cases, periodic, small $d n$, and large $d n$. The vertical axis is in linear scale. (b) The same as (a) but with the vertical axis being logarithmic scale. The horizontal axis in (a) and (b) represents the peak number. The numbers, 3, 4, 5, 6, and 7 , of the five peaks in the case of large $d n$ are arbitrarily assigned, being not relevant to the line shape recognition. To see the linear line shape, a straight line connecting two end points is drawn, respectively, in (a) and (b).

more localized, so that only five peaks are identified, seeing Figure 3 for their frequencies. The decrease of the number of spectra peaks is a consequence of larger $d n$ or steeper titled potential, because a few of WSL states (e.g., the farthest one) cannot be efficiently excited any more via the tunneling. Likewise, the spectra spacing of the peaks become wider with increasing $d n$, due to the larger eigenenergy differences between neighboring WSL states under steeper potential.

We have labeled the frequency values of all identified peaks from Figure 2(c) and plotted them in Figure 3. With the linearly scaled frequency axis, as shown in Figure 3(a), the frequency dots in the periodic case display a curved trend, consistent with the tight binding description of the miniband [27]. In contrast, the line shapes of the latter two cases can be approximately regarded as being linear. However, if the axis is switched to be logarithmic scale, seeing Figure 3(b), the frequency dots of the gradient cases are more close to a linear curve, especially for the large $d n$ case, where the dots of peaks 4,5 , and 6 fall completely on the straight line connecting two end points (the dots of peaks 3 and 7). The linear line shape in logarithmic scale reveals that these frequency values are actually in a geometric sequence, which is consistent with the analytical derivation.

From the experimental perspective, the critical requirement for observing the proposed WSL is to fabricate the dielectrics with designed refractive index $n_{j}=(1+d n)^{j}$. The feasible technique route of well-controlling refractive index or dielectric constant is to employ composite material whose permittivity can be fine-tuned by the component concentration according to effective medium theory [2931]. The composite suitable to our model may be the plastic foam where the porosity (air concentration) can be engineered to realize the desired dielectrics. We will conduct the experimental investigation along with this technique consideration in the future.

\section{Conclusions}

In conclusion, by making an analogy to the quantum counterpart and assuming the translational properties of the solution $F(x)$, we have shown the photonic WSL in the system of coupled EM cavities, where the tilted potential effect is mimicked by imposing the gradient variation of refractive index along the propagation direction, may have the eigenenergies (frequencies) in geometrical progression. Within the approximation of small gradient, the WSL states have the equidistant frequency spacing in the spectrum. The numerical simulations of the model system, the stack of structured plates, have illustrated the generalized photonic WSL state with geometrically progressed energies.

\section{Conflict of Interests}

The authors declare that there is no conflict of interests regarding the publication of this paper.

\section{Acknowledgments}

This work was supported by the National Natural Science Foundation of China (Grant nos. 11104198 and 11474212), the Natural Science Foundation of Jiangsu Province (Grant no. BK20141191), and a Project Funded by the Priority Academic Program Development (PAPD) of Jiangsu Higher Education Institutions. The authors thank Professors Gang Wang and Zhi Hong Hang for the beneficial discussions. 


\section{References}

[1] J. D. Joannopoulos, R. D. Meade, and J. N. Winn, Photonic Crystals: Molding the Flow of Light, Princeton University Press, Princeton, NJ, USA, 1995.

[2] G. H. Wannier, "Dynamics of band electrons in electric and magnetic fields," Reviews of Modern Physics, vol. 34, pp. 645655, 1962.

[3] E. E. Mendez and G. Bastard, "Wannier-stark ladders and bloch oscillations in superlattices," Physics Today, vol. 46, no. 6, pp. 34-42, 1993.

[4] G. Monsivais, M. del Castillo-Mussot, and F. Claro, "Starkladder resonances in the propagation of electromagnetic waves," Physical Review Letters, vol. 64, no. 12, pp. 1433-1436, 1990.

[5] R. Sapienza, P. Costantino, D. Wiersma, M. Ghulinyan, C. J. Oton, and L. Pavesi, "Optical analogue of electronic Bloch oscillations," Physical Review Letters, vol. 91, no. 26, Article ID 263902, 2003.

[6] V. Agarwal, J. A. Del Río, G. Malpuech et al., "Photon bloch oscillations in porous silicon optical superlattices," Physical Review Letters, vol. 92, no. 9, Article ID 097401, 2004.

[7] J. O. Estevez, J. Arriaga, A. Mendez-Blas, E. Reyes-Ayona, J. Escorcia, and V. Agarwal, "Demonstration of photon Bloch oscillations and Wannier-Stark ladders in dual-periodical multilayer structures based on porous silicon," Nanoscale Research Letters, vol. 7, article 413, 2012.

[8] G. Malpuech, A. Kavokin, G. Panzarini, and A. Di Carlo, "Theory of photon Bloch oscillations in photonic crystals," Physical Review B: Condensed Matter and Materials Physics, vol. 63, no. 3, Article ID 035108, 2001.

[9] P. B. Wilkinson, "Photonic Bloch oscillations and WannierStark ladders in exponentially chirped Bragg gratings," Physical Review E: Statistical, Nonlinear, and Soft Matter Physics, vol. 65, Article ID 056616, 2002.

[10] V. Lousse and S. Fan, "Tunable terahertz Bloch oscillations in chirped photonic crystals," Physical Review B, vol. 72, no. 7, Article ID 075119, 2005.

[11] G. Wang, J. P. Huang, and K. W. Yu, "Tunable photonic Bloch oscillations in electrically modulated photonic crystals," Optics Letters, vol. 33, no. 19, pp. 2200-2202, 2008.

[12] X. Kang and Z. Wang, "Optical Bloch oscillation and resonant Zener tunneling in one-dimensional quasi-period structures containing single negative materials," Optics Communications, vol. 282, no. 3, pp. 355-359, 2009.

[13] T.-B. Wang, C.-P. Yin, W.-Y. Liang, and H.-Z. Wang, "Bloch oscillations in one-dimensional photonic crystal coupled microcavity composed of single-negative materials," Physics Letters A, vol. 373, no. 45, pp. 4197-4200, 2009.

[14] T.-B. Wang, N.-H. Liu, X.-H. Deng, and Q.-H. Liao, "Bloch oscillations in one-dimensional coupled multiple microcavities containing negative-index materials," Journal of Optics, vol. 13, no. 9, Article ID 095705, 2011.

[15] Z.-H. Li, X.-N. Pang, J.-W. Dong, and H.-Z. Wang, "Electromagnetic Bloch-like oscillations in one-dimensional quasicrystal consisting of negative permeability metamaterial," Europhysics Letters, vol. 95, no. 3, Article ID 36004, 2011.

[16] T.-B. Wang, N.-H. Liu, T.-B. Yu, X.-H. Deng, X.-M. Xu, and Q.H. Liao, "Electromagnetic Bloch oscillation in one-dimensional multiple microcavities composed of metamaterials," Chinese Physics B, vol. 23, no. 4, Article ID 044101, 2014.
[17] U. Peschel, T. Pertsch, and F. Lederer, "Optical Bloch oscillations in waveguide arrays," Optics Letters, vol. 23, no. 21, pp. 1701-1703, 1998.

[18] W. H. Lin, X. Zhou, G. P. Wang, and C. T. Chan, "Spatial Bloch oscillations of plasmons in nanoscale metal waveguide arrays," Applied Physics Letters, vol. 91, no. 24, Article ID 243113, 2007.

[19] A. Joushaghani, R. Iyer, J. K. S. Poon et al., "Quasi-bloch oscillations in curved coupled optical waveguides," Physical Review Letters, vol. 103, no. 14, Article ID 143903, 2009.

[20] G. Corrielli, A. Crespi, G. Della Valle, S. Longhi, and R. Osellame, "Fractional Bloch oscillations in photonic lattices," Nature Communications, vol. 4, article 1555, 2013.

[21] J. Mateos and G. Monsivais, "Stark-ladder resonances in elastic waves," Physica A: Statistical Mechanics and Its Applications, vol. 207, no. 1-3, pp. 445-451, 1994.

[22] L. Gutiérrez, A. Díaz-De-Anda, J. Flores, R. A. MéndezSánchez, G. Monsivais, and A. Morales, "Wannier-Stark ladders in one-dimensional elastic systems," Physical Review Letters, vol. 97, no. 11, Article ID 114301, 2006.

[23] H. Sanchis-Alepuz, Y. A. Kosevich, and J. Sánchez-Dehesa, "Acoustic analogue of electronic bloch oscillations and resonant zener tunneling in ultrasonic superlattices," Physical Review Letters, vol. 98, no. 13, Article ID 134301, 2007.

[24] M. M. de Lima, Y. A. Kosevich, P. V. Santos, and A. Cantarero, "Surface acoustic bloch oscillations, the wannier-stark ladder, and landau-zener tunneling in a solid," Physical Review Letters, vol. 104, no. 16, Article ID 165502, 2010.

[25] C. Kittel, Introduction to Solid State Physics, John Wiley \& Sons, New York, NY, USA, 1996.

[26] W. J. Wen, L. Zhou, B. Hou, C. T. Chan, and P. Sheng, "Resonant transmission of microwaves through subwavelength fractal slits in a metallic plate," Physical Review B, vol. 72, no. 15, Article ID 153406, 2005.

[27] B. Hou, X. Xiao, and W. Wen, "Photonic bands from cascaded metallic plates with subwavelength slits," Journal of Optics, vol. 14, no. 5, Article ID 055102, 2012.

[28] Simulations were performed using the commercial package QuickWave developed by QWED, Poland, http://www.qwed .com.pl.

[29] W. Cai and V. M. Shalaev, Optical Metamaterials: Fundamentals and Applications, Springer, New York, NY, USA, 2010.

[30] Z. L. Mei, J. Bai, and T. J. Cui, "Gradient index metamaterials realized by drilling hole arrays," Journal of Physics D: Applied Physics, vol. 43, no. 5, Article ID 055404, 2010.

[31] H. Han, L. Wu, X. Tian, D. Li, M. Yin, and Y. Wang, "Broadband gradient refractive index planar lens based on a compound liquid medium," Journal of Applied Physics, vol. 112, no. 11, Article ID 114913, 2012. 

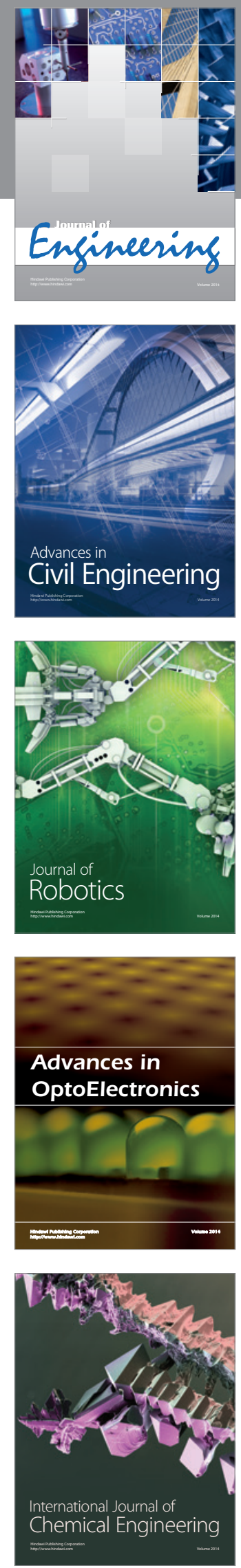

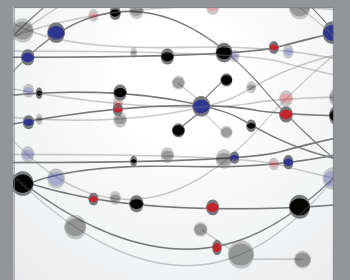

The Scientific World Journal
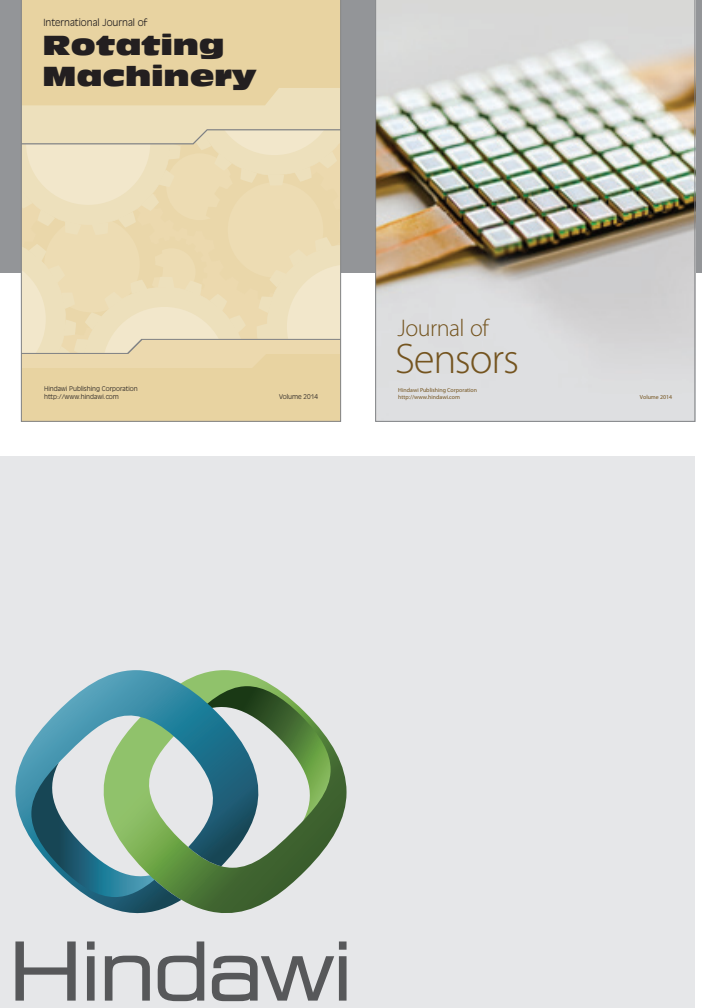

Submit your manuscripts at http://www.hindawi.com
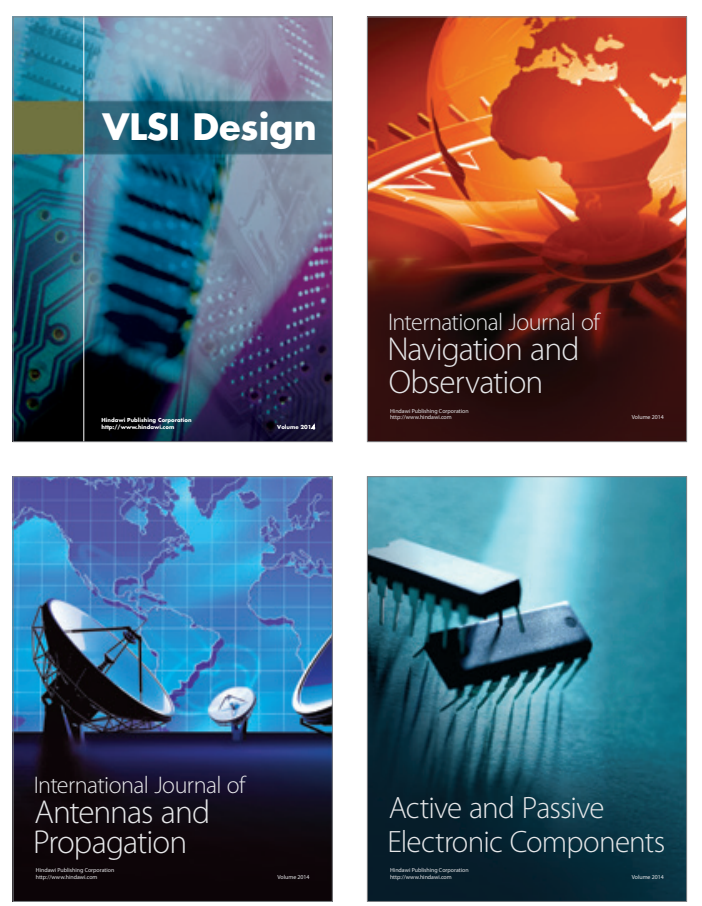
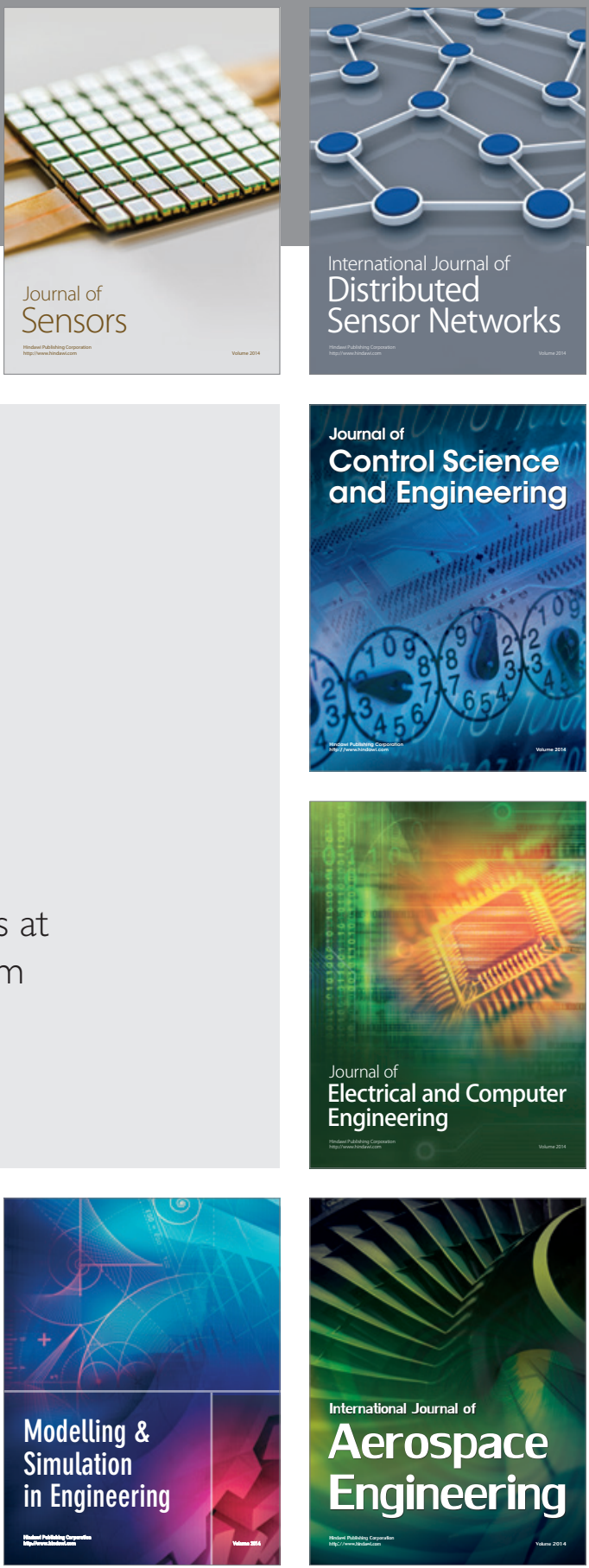

Journal of

Control Science

and Engineering
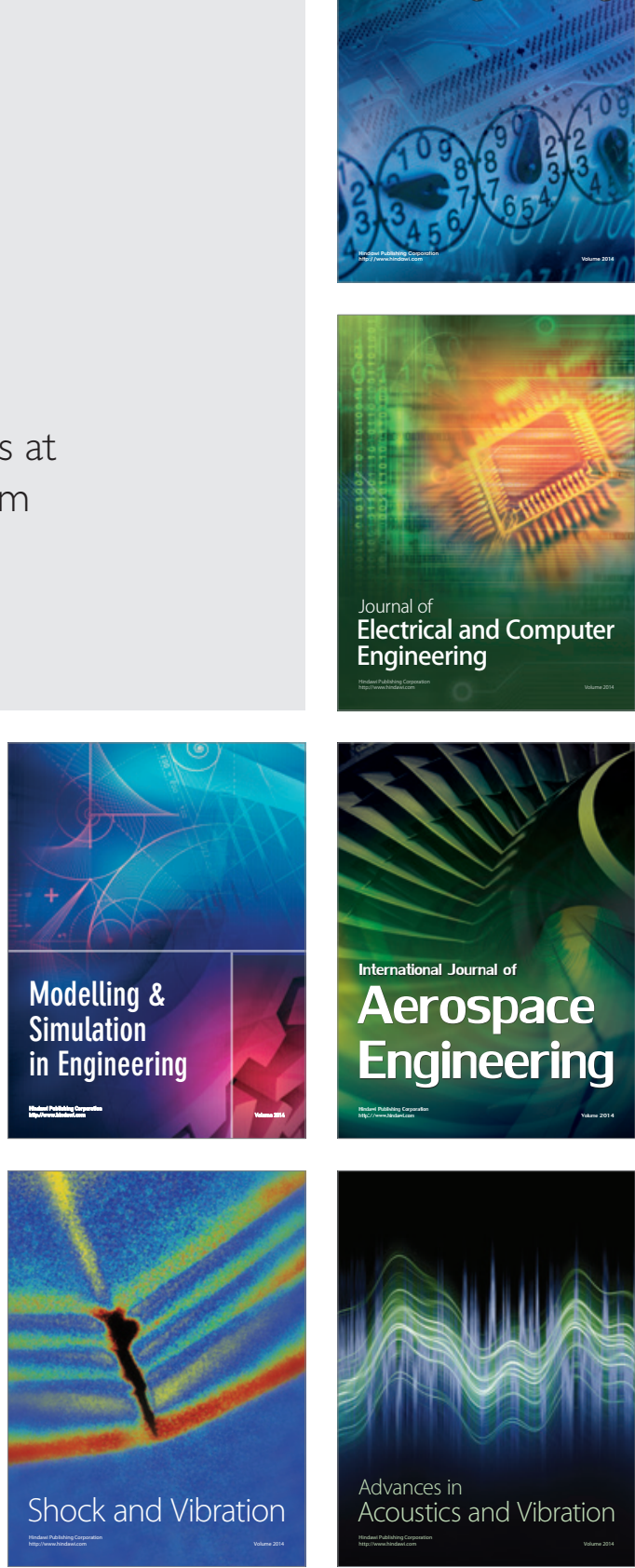